\title{
El "triángulo mágico" en el III Plan: Pleno empleo, estabilidad de precios y crecimiento económico
}

\author{
POr JAVIER GOROSQUIETA*
}

Un supremo ideal de la política de desarrollo-ideal prácticamente inalcanzable en un 100 por 100--es el de conseguir simultáneamente este triple objetivo: pleno empleo del trabajo nacional disponible, fuerte tasa de crecimiento de la renta nacional y estabilidad de los precios. Es éste el "triángulo mágico" del que hablan algunos economistas.

El texto del III Plan de Desarrollo es plenamente consciente de la dificultad de alcanzar totalmente y a la vez esa triple meta. Así de matizadas son, en primer lugar, sus declaraciones de propósito al respecto. Dice sobre el pleno empleo, como aspiración del III Plan: "El pleno empleo y la mejor utilización del trabajo constituyen objetivo fundamental de la politica de desarrollo socioeconómico. La creación de puestos de trabajo atenderá principalmente a la consecución del pleno empleo..." (pág. 145). Escribe sobre la tasa de crecimiento de la economía: "Diversas razones inducen a proponer, como objetivo al que debiera tenderse, el de un ritmo de crecimiento del Producto Nacional Bruto a precios de mercado del 7 por 100 anual acumulativo en términos reales" (pám gina 226). Y afirma sobre estabilidad de precios: “... todo proceso de crecimiento económico tiene una serie de cundicionantes. En primer lugar, ha de ser equilibrado, lo que implica evitar alzas excesivas de precios que, además de provocar injusticias distributivas, impiden una asignación eficiente de los recursos productivos, en perjuicio de un sano desarrollo económico a medio y largo plazo, y dan lugar, en fin, a una deterioración de precios con el exterior" (pág. 216).

\footnotetext{
* Doctor en Economía.
} 
Como vemos, ya en estas declaraciones sobre objetivos, el "triángulo mágico" se rompe claramente por el lado de la estabilidad. En efecto, no se habla de estabilidad de precios, sino de "evitar alzas excesivas" en los mismos. Por sú lado, el pleno empleo y el crecimiento anual acumulativo del 7 por 100 son dos cimas que se observan no con la seguridad de poderlas dominar, sino con la simple voluntad de tender a ellas, de subir por las laderas.

Pero veamos, a poder ser cifrados, los propósitos del III Plan sobre este triple objetivo:

\section{ESTABHLDAD DE PRECIOS}

1) Al hablar de las variables exógenas del modelo del III Plan, prevé el texto un crecimiento anual acumulativo, en términos reales del coste de la vida, entre un 3 y un 4 por 100 (pág. 221).

2) En el Cuadro Macroeconómico $1972-75$ se hacen, sobre el crecimiento de todas las macromagnitudes, dos tipos de previsiones: una, en términos reales o pesetas constantes, y otra, de mayor nivel, en términos monetarios (pág. 228):

\begin{tabular}{|c|c|c|}
\hline & \multicolumn{2}{|c|}{$\begin{array}{c}\text { Porcentaje de crecimiento anual } \\
\text { acumulativo }\end{array}$} \\
\hline & Términos reales & Términos monetarios \\
\hline Producto Nacional Bruto $\ldots \ldots \ldots \ldots$ & 7 & 11 \\
\hline Importaciones $\ldots \ldots \ldots \ldots \ldots \ldots \ldots \ldots$ & 11 & 15,5 \\
\hline $\begin{array}{lllllllll}\text { Consumo Privado } & \ldots & \ldots & \ldots & \ldots & \ldots & \ldots & \ldots\end{array}$ & 6,5 & 10,9 \\
\hline Consumo Público $\ldots \ldots \ldots \ldots \ldots \ldots \ldots \ldots$ & 5,3 & 12 \\
\hline Formación Bruta de Capital ... . . . . ... & 9,7 & 14 \\
\hline $\begin{array}{llllllllll}\text { Exportaciones } & \ldots & \ldots & \ldots & \ldots & \ldots & \ldots & \ldots & \ldots\end{array}$ & 10 & 14 \\
\hline
\end{tabular}

Todo esto significa que un cierto grado de inflación de los precios y, por lo tanto, de depreciación del dinero, está cficialmente programado en el texto del III Plan. En el mismo cuadro del texto vemos cómo los recursos disponibles en 1975 se calculan en 3.961 .800 millones de pesetas, en pesetas de 1971, y en 4.597.400 millones de pesetas, en moneda de 1975. Lo cual quiere decir que se cuenta desde el principio con una depreciación del dinero en torno al 16,3 por 100 durante el cuatrienio 1972-75.

\section{TASA DE CRECIMENTO}

No el 7 por 100 , porque, "en términos generales, la tasa de crecimiento del Producto Nacional Bruto estará comprendida entre el 6,5 y el 7 por 100 anual acumulativo, siempre y cuando se cum- 
plan las hipótesis sobre la evolución de las variables exógenas antes indicadas» (III Plan, pág. 223).

\section{PLENO EMPLEO}

En sus directrices de política económica, el III Plan no sólo aspira al pleno empleo, sino que, respecto de los trabajadores emigrantes en el extranjero, dice: "Asimismo se facilitará su reinstalación laboral y social en España” (pág. 148).

En cifras concretas, se aspira a la creación de 480.100 nuevos puestos de trabajo en la industria y 324.400 en el sector servicios; esto supone un ritmo del 4,1 por 100 anual acumulativo en la creación de nuevos puestos de trabajo (Cfr. pág. 224).

De ese 4,1 por 100 , el 2 por 100 será necesario para colocar las 283.700 personas actualmente activas en el sector primario que se espera lo abandonarán durante el cuatrienio 1972-75. El 2,1 restante queda para atender al crecimiento neto de la población activa española.

Si estas previsiones fueran correctas, ol pleno empleo podría quedar asegurado, puesto que el aumento de la población total española se calcula en un 1 por 100 anual acumulativo, y la expe. riencia de la década 1960-70 demuestra que el aumento de la población activa en España no ha sido tampoco superior al 1 por 100 anual. Habría, pues, lugar incluso para una inmigración neta de trabajadores españoles en otros países.

Pero ¿son exactas esas previsiones? No es fácil, naturalmente, averiquarlo, y habría que dar crédito a los redactores del III Plan si un organismo tan autorizado como la O. C. D. E., en su último informe sobre la economía española no hubiera puesto en este punto una sombra de duda. Dice, p. ej., este informe: "Una tasa de expansión del 7 por 100 no será, sin duda, suficiente para mantener el pleno empleo sin que más trabajadores continúen emigrando al extranjero... Dadas las previsiones relativas a la balanza de pagos, puede preguntarse si es bueno que España continúe exportando una parte de su ahorro nacional, mientras que una parte de su población activa sale del país". Y en otro lugar indica el informe que las previsiones macroeconómicas del III Plan implican una emigración neta anual de cuarenta y cinco mil trabajadores, si se quiere mantener el paro por debajo del 2 por 100 de la población activa española.

Por lo tanto, y resumiendo, la estabilidad de precios programa" da es sólo relativa, la tasa de crecimiento prevista es más bien algo inferior al 7 por 100 anual, y no parece fácil se pueda mante- 
ner un paro por debajo del 2 por 100 de la población activa, aun contando con la válvula de la emigración al exterior.

El III Plan, pues, aunque optimista en relación con el informe de la O.C.D. E, tiene conciencia de este precario equilibrio y relativiza así sus aspiraciones: "... el objetivo de crecimiento fijado podrá alcanzarse manteniendo la compatibilidad entre los tres objetivos básicos para el logro de la estabilidad interna y externa: mantenimiento de una tasa de desempleo inferior al 2 por 100 de la población trabajadora, alzas del índice de precios del Producto $\mathrm{Na}$ cional Bruto de un 3,7 por 100 anual y equilibrio exterior" (página 226).

\section{INFLACION MODERADA Y EGTINULANIE}

¿Qué pensar, pues, de la politica de pleno empleo, estabilidad relativa de los precios y tasa de crecimiento del PNP, tal como se encuentran planteadas en el III Plan?

En primer lugar, creo se puede aseverar que un moderado grado de inflación es un precio razonable si con él se lograra realmente, junto con el pleno empleo y una elevada tasa de crecimiento de la renta nacional, una mejora en la redistribución personal de los ingresos.

El economista inglés Dennis Robertson es quizás quien ha se. nalado más claramente los posibles efectos beneficiosos de una suave y permanente subida de los precios, "El nivel de precios suavemente creciente-dice-, en tanto no resulte tan abrupto como para generar el desorden social o minar los fundamentos del contrato.... estimula la producción de bienes, al beneficiar a los bolsillos de quienes controlan la industria, estimula asimismo sus energías y actividades..., y este acicate a la producción, al añadirse al flujo de bienes, sirve para moderar el mismo aumento de precios que fue su primer origen". De ahí que "mientras el control de la producción esté en manos de una minoría, recom. pensada por medio de un beneficio variable, es posible que un nivel de precios suavemente creciente produzca, de hecho, los mejores resultados imaginables, no sólo para ellos, sino para toda la comunidad en su conjunto. $Y$ es posiblemente cierto que un nivel de precios en permanente descenso, aun por la mejor de las razones, demostraría ser deficiente en esos estímulos sobre los cuales la sociedad moderna, prudentemente o no, ha descansado hasta ahora principalmente para mantener a sus miembros en pleno empleo y llevar adelante el trabajo que le tocaba realizar" (1).

(1) Citado por N. Kaldor en Ensayos sobre Política Económica. Edit. Tecnos, 1971, p. 216. 
Otro gran economista inglés, Nicholas Kaldor, se expresa asi, del mismo modo: "Dada una tasa lo bastante alta de productividad, y dado un adecuado suplemento en forma de inflación de la tasa real de crecimiento, no hay razón para que la prosperidad no haya de ser perpetua... De ahí que una tasa lenta y continua de inflación proporcione la ayuda más poderosa para obtener una permanente tasa de progreso económico" (2).

Estos dos economistas citados no son, desde luego, dos músi$\cos$ que desafinan en el conjunto de la orquesta. Hay toda una orquesta de economistas que abundan en las mismas ideas sobre los posibles efectos beneficiosos de una inflación moderada.

Se me podrá decir en este punto concreto que no es por ahí precisamente por donde existe el mayor riesgo en la marcha de la economía española. Se me podrá recordar que, según cifras oficiales, el 32 por 100 de la oferta monetaria disponible durante el II Plan fue absorbida por un incremento puramente inflacio. nario de los precios. Se me dará, p. ej., la cifra de la subida del coste de la vida en más de un 9 por 100 durante 1971.

Es cierto: la falta de inflación suficiente no ha sido precisamente el punto flaco de la economía española durante los últimos diez años. Pero esto no impide el que se pueda seguir afirm mando, en teoría, que un moderado grado de inflación, bien administrado, puede ser un apetecible estimulante en el desarrollo. $Y$ que, por lo tanto, no puede ser condenada en sí misma la moderada tasa de crecimiento de los precios programada en el fll Plan de Desarrollo, si favorece el logro del pleno empleo y una prosperidad no discriminatoria.

Lo malo sería pagar el precio de la inflación moderada, sin obtener ninguno de sus posibles efectos beneficiosos. Lo malo sería, p. ej., la presencia simultánea de inflación y estancamiento económico; es ésta la famosa "stangflación", que se puede dar y que ha padecido de hecho la economía española durante 1971.

\section{CONDICIONES DE UNA INFLACION ESTIMUEANTE $\checkmark$ NO DISCRIMINATORIA}

¿En qué condiciones, pues, una inflación moderada puede resultar tanto estimulante como equitativa? Para que resulte estimulante es preciso:

- Primero, que exista un clima empresarial no enrarecido por la inseguridad, el desconcierto y la desconfianza sobre los propósitos de la política económica a medio y largo plazo. Un sis.

(2) N. Kaldor, o, c, p. 215. 
tema de libertad de empresa, como es el propio de las economias no colectivistas, es movido por la iniciativa del empresario privado como motor principal, aunque acompañado, eso sí, por el intervencionismo creciente del Estado. Ese motor principal necesita cierta garantía de futuro; si el empresario privado desconfía un tanto radicalmente, de nada servirán las clásicas medidas reactivadoras, como lo ha demostrado-repito-la experiencia de la economía española en 1971. En este último supuesto no hay otro camino que restablecer ( sa confianza perdida, a no ser que se quiera intentar la otra vía de socialización total de la actividad económica productiva.

- Segundo, que ese incremento moderado de los precios se refleje, como sucederá normalmente, en un aumento de los beneficios de las empresas, a través de la subida progresiva de los volúmenes de producción y de ventas. A su vez, como indicaba Robertson, este aumento progresivo de la oferta de bienes debe hacer de freno en el alza de los precios, de tal manera que éstos no erosionen, sino en moderada medida, el poder adquisitivo del dinero.

Pero no es suficiente que la inflación moderada sea un acicate al desarrollo por la mejora de las expectativas empresariales. Además de eso, la inflación moderada debe ser compatible con los postulados de la equidad. De estos últimos, el más im. portante es el que se refiere a los perceptores de rentas fijas, de rentas relativamente congeladas, como son los salarios, y también los que atañen a los pequeños ahorradores.

Es bien sabido que la inflación detrae poder de compra a los beneficiarios de rentas fijas, como son los pensionistas y los propietarios de viviendas con alquileres congelados. Si se programa, por lo tanto, algún grado de inflación, es necesario prever al mismo tiempo un sistema de actualización de las pensiones y una cierta, suficiente, liberalización de los alquileres congelados. De lo contrario, la programación del desarrollo sería claramente discriminatoria, a favor de los empresarios y en contra de tales beneficiarios de rentas fijas o congeladas.

Los salarios, en tanto no están sometidos en la práctica a un continuo proceso de revisión, participan relativamente del carácter de rentas fijas o congeladas. Por lo mismo, en tanto permanecen invariables mientras los precios suben, va disminuyendo su participación en el pastel de la renta nacional.

Los pequeños ahorradores son también perjudicados. Como escribe T. F. Durnburg, "bonos del Gobierno, pólizas de seguro, depósitos de ahorro y otras formas de activos productores de interés fijo, que atraen a los pequeños ahorradores, se convierten 
realmente en otras tantas trampas, donde el valor de los ahorros es corroído por la elevación de nivel de precios" (3).

O, según Samuelson, "los bonos del Gobierno de los Estados Unidos, comprados a 75 dólares en 1948 , se reembolsan a 100 dólares en 1958; pero los 100 dólares de 1958 tenían un poder adquisitivo inferior a los 75 de 1948 ", por causa de la inflación.

\section{PRESENCIA DE AOUELLAS CONDICIONES EN EL III PLAN}

¿Están previstas en el III Plan las condiciones necesarias para que la moderada inflación programada resulte tanto estimulante como equitativa? Veamos.

\section{Confianza empresarial}

Seguramente que las dos causas más importantes que han consumido la confianza del empresariado español durante estos últimos años han sido, en primer lugar, la marcha pendular de nuestra economía en los últimos años, el brusco y rítmico stop and go, frenazo y aceleración, provocado por la política económi$c a, y$, en segundo lugar, la inseguridad de nuestra integración en la Comunidad Económica Europea.

¿Adopta el III Plan una postura en torno a estos dos extre. mos? En cuanto a la elevación de la moral del empresario, el hecho mismo de que se publique ese programa de política económica a medio plazo, que es un Plan de Desarrollo, debería contribuir considerablemente a dar mayor seguridad al empresario privado en sus decisiones; el Plan le ofrece, clarificado, el marco de la política económica durante cuatro años, marco en el que el empresario debe asumir el riesgo de empresa. Pero sucede que la existencia de los dos Planes anteriores no fue óbice para esa marcha ondulante y sorpresiva, a que antes aludiamos, de la economía. No parece, por lo tanto, que el texto del III Plan vaya a tener en sí mismo una fuerza tonificante definitiva. $Y$ esto a pesar de que el III Plan parece haber aprendido en este punto de sus dos predecesores. "Sigue siendo preocupación fundamental - dice-el lograr el mayor ritmo de crecimiento, compatible con la estabilidad interior y exterior de la economía, ya que, de no mantenerse los equilibrios fundamentales, el desarrollo se vería sometido a movimientos pendulares que es preciso evitar" (página 141). Pero ¿quién se fía de palabras? Será la consistencia y congruencia de la propia política económica del Estado la única

(3) T. F. Dernburg: Macroeconomía. Ed. Revista de Derecho Privado. Madrid, 1962, p. 302 . 
que pueda devolver al empresario la confianza titubeante o medio perdida. Y esa consistencia no se puede demostrar en unos pocos días o en unas pocas semanas.

Respecto de nuestra integración en la Comunidad Económica Europea, el III Plan se muestra necesariamente cauto y condicional. Porque esa integración no depende sólo de nosotros, sino también de nuestros interlocutores europeos. "Es presumible pensar-dice el texto del Plan-en una evolución ulterior como consecuencia de la integración de la economia española en áreas supranacionales, si finalmente se lleva a tërmino. Este proceso aparece, en principio, determinado con la entrada en vigor del Acuerdo Preferencial con la Comunidad Económica Europea" (página 36). "... Hay que preparar-continúa-con la debida antelación nuestra estrategia ante eventualidades tales como la incorporación de nuevos miembros" a la C.E.E. (pág. 37). Efectivamente la ampliación de la Europa de los "Seis" a la Europa de los "Diez" es ya un hecho. $Y$ todo técnico está de acuerdo en que, con el ingreso en el Mercado Común de Inglaterra, Irlanda, Noruega y Dinamarca, nuestro Acuerdo Preferencial ha quedado ya totalmente envejecido y superado, al año y medio de su entrada en vigor. ¿Estará España dispuesta a pagar algún precio político por el logro de una mayor facilidad de acceso a la Comunidad Económica Europea? He aquí otro elemento de inseguridad.

Si nos fijamos en la segunda condición de que hablábamos para que la inflación resulte estimulante, es decir, que el aumento moderado de los precios se refleje en un incremento de los beneficios empresariales, se puede asegurar que se producirá ese efecto beneficioso para la empresa siempre que se trate de una clásica, aunque moderada, inflación de demanda, es decir, que a la subida de los precios responda adecuadamente el incremento de la producción y oferta de toda clase de bienes y servicios económicos. Por el contrario, inflación con estancamiento sería desastroso; la suma de todos los males, por así decirlo, sin mezcla de bien alguno. Pues bien, la subida de los precios, como expresión o efecto de un tirón de la demanda, mejora siempre las expectativas empresariales y es de esperar produzca el efecto deseado de incremento de producción y oferta, siempre que no se opongan obstáculos de mayor envergadura. Con lo cual volvemos sobre lo indicado anteriormente, sobre el descorazonamiento y apatía empresariales. Ahí es, por lo tanto, principalmente, donde habrá que dar la batalla; hay una inercia que parece inmovible con sólo el acicate de precios elevados.

\section{Condiciones de equidad}

En relación con las condiciones para que una inflación moderada resulte equitativa, nos referíamos a los perjuicios que la in- 
flación puede producir principalmente en tres grupos de personas: pensionistas y perceptores de renta fijas (alquileres), asalariados y pequeños ahorradores.

En cuanto a los pensionistas, la forma de neutralizar los efectos perniciosos de la inflación es clara: adoptar un sistema de actualización de las pensiones de acuerdo con la subida del coste de la vida. El texto del III Plan parece consciente de este problema "... se aprecia-dice-la insuficiencia de las pensiones, lo que es causa de desigualdad en las rentas y en la participación en los frutos del desarrollo y, a la vez, distorsiona la estructura adecuada de la población activa y dificulta la aceleración del proceso productivo, al forzar a permanecer en sus puestos de tran bajo a una mano de obra envejecida" (pág. 35). Y en otro lugar: "Se configurará un sistema de pensiones de retiro que tienda a la mayor homogeneidad de tales prestaciones en los distintos regímenes de la Seguridad Social, así como a su revalorización y actualización periódica, teniendo en cuenta los salarios percibidos por los trabajadores en activo y los superiores niveles de vida a que vaya accediendo la comunidad" (pág. 147). Al no encontrar en el texto cantidades concretas presupuestadas para esta actualización, sólo queda esperar que esos buenos deseos no se queden en simples declaraciones programáticas.

Sobre una relativa descongelación de antiguos alquileres, algo parece prometer el siguiente párrafo del III Plan: "Se revisará la vigente legislación de arrendamientos urbanos, con el fin de atender a la conservación del patrimonio iesidencial y de su adecuación funcional a las exigencias actuales de la demanda" (pág. 151).

\section{Los salarios}

Respecto del salario mínimo, no solamente se promete su puesta al día y revísión anual, sino el realizar un esfuerzo efectivo por pasar de la noción de salario mínimo a la de salario suficiente, de acuerdo con la normativa del Fuero del Trabajo. (Cfr. păg. 146 s., § 7.)

De la política salarial en general no se le podría pedir mayor perfección al planteamiento programático del III Plan. "La política de salarios-escribe-se coordinará con las medidas de política general de estabilidad de precios en el marco de una política de todos los precios y de todas las rentas. Esta política de rentas y precios se complementará con medidas fiscales apropiadas y de redistribución de la propiedad, así como las de promoción social ya mencionadas, a fin de lograr un reparto más equitativo en la renta nacional" (pág. 147). Punto fundamental de apoyo será la contratación colectiva, para cuyo perfeccionamiento se elaborará una nueva Ley de Convenios Colectivos (cfr. ibídem). 
No se puede pedir más como normativa general: sistemas flexibles de revisiones periódicas, a través principalmente de la contratación colectiva, dentro de un sistema coordinado de polí. tica de precios y de rentas. Y no sólo redistribución personal de los ingresos, por medio particularmente de la política fiscal progresiva, sino redistribución incluso de la propiedad. Como antes, sólo nos queda desear que esta redistribución así planteada de los ingresos y de la propiedad sea más eficaz y radical que en los diez años anteriores, dentro de una ininterrumpida prosperidad económica también para el empresario.

En otros países, en los que está permitida, aunque regulada, la huelga, por un lado, y el despido, por otro, las fuerzas del mercado libre determinan bastante fundamentalmente la política salarial. En una situación de pleno empleo aumenta el poder nego" ciador de los sindicatos obreros; y como la coyuntura de pleno empleo suele coincidir con la fase de prosperidad económica en general y del capital de las empresas en particular, los trabajadores asalariados suelen tener en ese momento casi todas las bazas a su favor: no hay obreros desocupados que los empre. sarios pudieran contratar a menores precios, las empresas pueden soportar una mayor carga por salarios, puesto que los beneficios son elevados, $y$, finalmente, las peticiones sindicales aparecen justificadas por la elevación del coste de la vida que acompaña normalmente a las etapas de prosperidad. En una situación de desempleo sucede todo lo contrario, ya que no se suelen dar ninguna de las condiciones anteriores.

En nuestro país las cosas cambian, porque ni la huelga está permitida, ni es libre el despido, sino circunscrito a circunstancias muy especiales de sanción o crisis, o, si se quiere, mediante una importante indemnización al trabajador asalariado.

En estas circunstancias se puede mantener una aparente si tuación de pleno empleo aun en fases de depresión económica, es decir, aun en momentos en que las empresas trabajan a niveles inferiores a los de su capacidad normal y en situaciones on que decrece la cartera de pedidos. Ciertamente no es ésta la coyuntura sostenida de pleno empleo que interesa lograr en el desarrollo; ese llamado "pleno empleo" disfraza un paro encubierto que implica falta de utilización normal de los recursos de mano de obra disponible. $Y$ el forzar en esas circunstancias la contratación colectiva, apoyándose en una coyuntura no económica, sino puramente institucional, de "pleno empleo", podría suponer el hundimiento injusto de algunas empresas y el estrechamiento excesivo de los beneficios de otras muchas más. Es pre ciso que el pleno empleo responda a la significación económica de tal fenómeno, y es entonces el momento de forzar todo lo que sea preciso la contratación salarial colectiva. Bienvenida sea, pues, una tasa moderada de inflación, si, manteniendo una pros. 
peridad permanente, mejora a la vez los beneficios empresariales y la fortaleza negociadora de los trabajadores asociados.

\section{El ahorro}

Hablábamos, finalmente, de un tercer grupo que sufre siempre el impacto perjudicial de la inflación: el grupo de los pequeños ahorradores que tal vez tienen su dinero depositado en una cuen. ta a plazo al 2 por 100 de interés y que la subida de los precios a un ritmo mayor le puede quitar año tras año parte del poder de compra de la cantidad que depositó.

La solución teórica parece clara: asegurar a los ahorradores sus depósitos y sus intereses, no en unidades monetarias o en tantos por ciento de unidades monetarias, sino en poder adquisitivo. Pero ¿quién le pone el cascabel al gato? No encontramos huella de nada de esto en el texto del III Plan, lo cual quiere decir que el Plan, al programer la inflación y no programar la permanencia, al menos, del poder de compra de los depósitos de ahorro, consiente implícitamente en el robo que la inflación va a perpetrar sobre los poderes adquisitivos depositados en cuen. tas de ahorro. Se podrá decir que apenas hay experiencias sobre el particular. Es cierto; pero para eso parece debería estar la imaginación de las nutridas listas de planificadores y colaboradores de la Comisaría del Plan. Convendrá esperar otra oportunidad y que la dinámica redistributiva con otros medios, el fiscal sobre todo, palíe en parte el asalto al poder de compra de los pequeños ahorradores.

\section{TASA DE CRECIMIENTO}

Del "triángulo mágico" descrito al principio: pleno empleo, es" tabilidad de precios y elevada tasa del crecimiento de la renta nacional, nos queda por aludir a este último. ¿Va a ser posible alcanzar la meta propuesta de crecimiento anual acumulativo del producto nacional bruto entre un 6.5 y un 7 por 100 en términos reales, es decir, en pesetas constantes? Según cifras oficiales, el crecimiento anual acumulativo y en términos reales fue del 6,2 por 100 en el I Plan, y del 6,7 por 100, en el II. El III Plan, pues, trata de superar en este objetivo clave a los dos anteriores. ¿Se ha tecnificado tanto la economía española durante estos últimos años; ha aumentado, además, tanto el nivel de formación profesional; han mejorado en tan alto grado y en tan poco tiempo las condiciones estructurales o básicas de nuestra economía de tal manera que se pueda esperar con optimismo la superación de los Plantes anteriores? No lo sabemos con seguridad, pero mucho me temo dos cosas, y con esto termino: primera, que su- 


\section{J. GOROSOUIETA}

cumbamos al triunfalismo de superar a cualquier precio los obje. tivos propuestos, como sucedió en el I plan, lo cual fue causa de su fracaso relativo; segunda, que esa tasa de crecimiento se logre a costa de abandonar relativamente las reformas estructurales de nuestra economía, que son las únicas que pueden esta. bilizarnos a largo plazo y poner las sólidas bases de una pros. peridad permanente. 